\section{Oberflächen-Tage bei} Sturm

A $m$ 4. und 5. Juli 2013 veranstaltet der Anlagenbauer Sturm, Salching, zum vierten Mal die Oberflächen-Tage. Die Veranstaltung ist integriert in die Open-House-Days-Woche, vom 1. bis 5. Juli, zu der Kunden und Interessenten einladen sind, die Sturm-Gruppe zu besichtigen. Auf den OberflächenTagen sind unter anderem Vorträge zu folgenden Themenbereichen geplant: Reinigung und Vorbehandlung - unter anderem mittels Plasma und $\mathrm{CO}_{2}-$, Lackierung und Lacke. Vorgesehen sind zudem Vorträge über nachwachsende Hochleistungskunststoffe, die bereits industriell genutzt und auch lackiert werden, sowie über biogene Polymere. Auf der Veranstaltung wird live eine Plasmabeschichtungsanlage für Kunststoffe vorgestellt. Darüber hinaus sind Erfahrungsberichte von Anlagenbetreibern geplant.

Weitere Informationen unter www.sturm-gruppe.com

\section{Termine}

Guangzhou (China)

21.-23.5.2013

SF Expo China 2013

Veranstalter: Wise Exhibition

(Guangdong) Co.,Ltd

www.sf-expo.cn

Tel. $+20-37599008$

Braunschweig

12.-13.6.2013

CVD Beschichtungstechnologien

Veranstalter: Otti

www.otti.de

Tel. 0941 29688-11

Reutlingen

4.-5.7.2013

Elektrostatik verstehen und sicher beherrschen

Veranstalter: Schnier Elektrostatik $\mathrm{GmbH}$

www.schnier-elektrostatik.de Tel. 07121 90973-63

\title{
General Motors ernennt BASF zum Lieferanten des Jahres 2012
}

7 um neunten Mal innerhalb der vergangenen elf Jahre wurde BASF von General Motors (GM) zum Lieferanten des Jahres ausgezeichnet. Beate Ehle, President Market and Business Development BASF Nordamerika, und Joe Schmondiuk, BASF Vice President Global Accounts OEM Coatings Solutions, nahmen die Auszeichnung stellvertretend für den Unternehmensbereich Coatings bei GM's jährlicher Verleihung am 13. März 2013 entgegen. Der Unternehmensbereich Coatings der BASF unterstützt GM mit modernen Lackierprozessen sowie seinem Know-how bei Effektpigmenten und Technologien. „BASF spielte eine entscheidende Rolle für den GM-Erfolg im vergangenen Jahr. Ihr Einsatz und das Engagement übertreffen unsere Erwartungen in Bezug auf Innovationen, Lieferung von qualitativ hochwertigen Produkten und Services sowie einer herausragenden Wertschaffung", sagte Grace Lieblein, GM Vice President, Global Purchasing und Supply Chain.

\section{Bei der \\ Preisverleihung (von links): Randy Pappal, Executive Director \\ Global Exterior Purchasing GM; Joseph Schmondiuk, Vice President Global Accounts BASF; \\ Beate Ehle, President Market and Business Development BASF; Greg Warden, Executive Director Global Engineering Body/Exterior GM}

\section{O\&S: Deutsche Messe jetzt alleinige Veranstalterin}

Do ie internationale Fachmesse für Oberflächen und Schichten, O\&S, wird künftig allein von der Deutschen Messe ausgerichtet. Sie bleibt aber weiterhin auf dem Stuttgarter Messegelände. Eine entsprechende Vereinbarung unterzeichneten Vertreter der Deutschen Messe und der Messe Stuttgart am Donnerstag in Stuttgart. Seit 2008 wur- de die O\&S gemeinsam von der Deutschen Messe und der Messe Stuttgart veranstaltet. 2014 dauert die O\&S vom 24. bis zum 26. Juni. Die Terminverschiebung war laut Deutsche Messe AG nötig geworden, da auch die beiden Parallelveranstaltungen aus dem Jahr 2012 - die Lasys und die Automotive Expo - diesen Termin gewählt hatten.

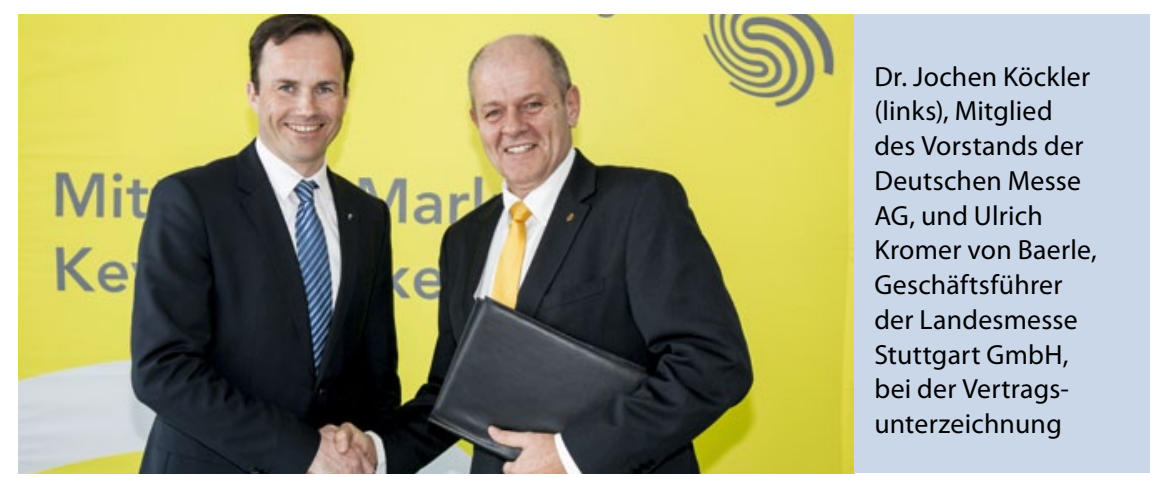

